\title{
II monitoraggio della terapia nel paziente con sclerosi multipla
}

\section{Lucia Moiola}

Coordinatore d'area ambulatori e DH, Centro Sclerosi Multipla, Ospedale San Raffaele, Milano

La scelta della terapia nella sclerosi multipla (SM) è guidata da un attento bilancio tra l'efficacia del trattamento nel prevenire le ricadute, la disabilità, l'attività neuroradiologica e l'atrofia, e il peso della terapia stessa in termini di sicurezza, tollerabilità e monitoraggio.

Negli ultimi venti anni, grazie ai progressi della ricerca clinica e di base, si è assistito all'introduzione di numerosi nuovi farmaci, ciascuno con uno specifico profilo rischio-beneficio.

Mentre per il glatiramer acetato (GA) e gli interferoni beta (IFN $\beta$ ), utilizzati nella SM da quasi venti anni, possiamo parlare di sicurezza a lungo termine, per i farmaci di più recente approvazione non tutti i rischi potenziali sono ancora noti. La finalità di questo capitolo è proprio quella di riassumere per ogni farmaco

\section{Indirizzo per la corrispondenza:}

Lucia Moiola

e-mail: moiola.lucia@hsr.it

Accettato: 30/11/2015 - Pubblicato online: 18/12/2015

(C) 2015 The Authors. This article is published by HPS Srl and licensed under Creative Commons Attribution-NC-ND 4.0 International (CC BY-NC-ND 4.0). Any commercial use is not permitted and is subject to Publisher's permissions. Full information is available at www.aboutpharma.com/publishing/riviste/aboutopen/ approvato i potenziali eventi avversi (EA), il monitoraggio per individuarli, la loro gestione e quando sia necessario interrompere la terapia (tp) in atto.

Iniziamo ad analizzare i farmaci in ordine di immissione in commercio (Tabella 1). Come osservazione generale va ricordato che, prima di iniziare qualsiasi trattamento, è opportuno eseguire esami ematochimici (EE) di base: emocromo, funzionalità renale, epatica e tiroidea in modo tale che nei controlli successivi eventuali alterazioni degli stessi siano più facilmente correlabili al farmaco intrapreso.

\section{Terapie sottocutanee (sc)}

Sono rappresentate da IFN e GA, farmaci immunomodulanti [1] che condividono la via di somministrazione sc. In relazione a tale modalità di somministrazione vi possono essere reazioni a livello del sito di iniezione che variano da eritema localizzato a rara necrosi cutanea [2-4]. Una corretta tecnica di iniezione e la rotazione del sito di iniezione sono fondamentali per ridurre questi effetti. Inoltre, la terapia con GA a lungo termine può causare lipoatrofia a livello dei siti di iniezione [4]. 
Tabella 1. Eventi avversi correlati ai DMD: possibili strategie per ridurli e monitoraggio

\begin{tabular}{|c|c|c|}
\hline DMD & Evento avverso o rischio & Strategie/monitoraggio \\
\hline \multirow[t]{4}{*}{ IFN-beta } & Reazioni a livello del sito di iniezione & Educazione del pz, tecnica e rotazione sito di iniezione \\
\hline & $\begin{array}{l}\text { Sintomi simil-influenzali prima o al } \\
\text { momento o anche dopo l'iniezione }\end{array}$ & FANS o antipiretici prima o al momento dell'iniezione \\
\hline & Leucopenia & $\begin{array}{l}\text { Esame basale e monitoraggio emocromo con FL, eventuale riduzione } \\
\text { frequenza e dosaggio }\end{array}$ \\
\hline & Alterazione AST/ALT & Esame basale e monitoraggio AST/ALT ${ }^{(1)}$ \\
\hline \multirow[t]{2}{*}{ GA } & Reazioni a livello del sito di iniezione & Educazione del pz, tecnica e rotazione sito di iniezione \\
\hline & $\begin{array}{l}\text { Reazione sistemica immediata post- } \\
\text { iniezione }\end{array}$ & Educazione del pz e rassicurazione \\
\hline \multirow[t]{3}{*}{ MTX } & Nausea/vomito & Antiemetici prima della somministrazione e poi al bisogno \\
\hline & Cardiotossicità & $\begin{array}{l}\text { Controllo ECG a ogni somministrazione; ecografia con FE basale e ogni } 3 \\
\text { cicli. Non superare dose di } 120 \mathrm{mg} / \mathrm{m}^{2}\end{array}$ \\
\hline & LMA & $\begin{array}{l}\text { Controllo emocromo con FL basale e monitoraggio. Non superare dose di } \\
60 \mathrm{mg} / \mathrm{m}^{2}\end{array}$ \\
\hline \multirow[t]{4}{*}{ NAT } & Reazioni infusionali & Riduzione della velocità d'infusione \\
\hline & Reazione da ipersensibilità & $\begin{array}{l}\text { Sospensione dell'infusione; somministrazione di corticosteroidi, antistaminici } \\
\text { e/o adrenalina }\end{array}$ \\
\hline & Aumento degli enzimi epatici & Esame basale e monitoraggio AST/ALT ${ }^{(1)}$ \\
\hline & PML & $\begin{array}{l}\text { STRATIFY basale: se negativo, ripetere ogni } 6 \text { mesi; se positivo, valutare } \\
\text { rischio e discuterne con il pz } \\
\text { Se STRATIFY negativo ripetere RM ogni 6-12 mesi, se STRATIFY positivo RM } \\
\text { ogni 3-4 mesi dopo i } 18-24 \text { mesi } \\
\text { Al minimo sospetto clinico o alla RM di PML, interruzione di NAT }\end{array}$ \\
\hline \multirow[t]{5}{*}{ FTY } & Leucopenia e linfopenia & Esame basale e monitoraggio. Sospensione se GB $<2000 / \mathrm{mm}^{3}$ o lym $<200 / \mathrm{mm}^{3}$ \\
\hline & Aumento degli enzimi epatici & Esame basale e monitoraggio AST/ALT ${ }^{(1)}$ \\
\hline & Infezioni erpetiche & $\begin{array}{l}\text { Screening basale: solo pz con anticorpi anti-VZV può iniziare FTY, altrimenti } \\
\text { occorre vaccino per VZV. Se sospetto di infezione trattamento immediato } \\
\text { con aciclovir, se infezione grave sospendere anche FTY }\end{array}$ \\
\hline & Edema maculare & $\begin{array}{l}\text { Consigliato esame basale e mandatorio esame di controllo a } 3 \text { mesi con FO } \\
\text { ed eventualmente OCT. Se sospetto edema: sospensione FTY }\end{array}$ \\
\hline & Eventi cardiaci (bradicardia, BAV, aritmia) & $\begin{array}{l}\text { ECG basale. Monitoraggio per } 6 \text { ore alla prima somministrazione: se ultima } \\
\text { FC rilevata è la più bassa del monitoraggio prolungare osservazione di altre } 2 \\
\text { ore; prolungare monitoraggio sino al mattino seguente se: FC }<45 \mathrm{bpm} \text {; BAV } \\
\text { III grado; BAV II grado di nuova insorgenza e intervallo QTc } \geq 500 \mathrm{msec}\end{array}$ \\
\hline \multirow[t]{3}{*}{ Teriflunomide } & Aumento degli enzimi epatici & $\begin{array}{l}\text { Esame basale e monitoraggio AST/ALT ogni } 2 \text { settimane per i primi } 6 \text { mesi e } \\
\text { quindi ogni } 8 \text { settimane. Se AST/ALT >3 volte LSN confermato, sospensione } \\
\text { del farmaco }\end{array}$ \\
\hline & Riattivazione della TBC & $\begin{array}{l}\text { Screening TBC, se positivo farmaco non va iniziato; se positivo durante } \\
\text { trattamento: sospensione teriflunomide, eliminazione con colestiramina e } \\
\text { trattamento per TBC }\end{array}$ \\
\hline & Teratogenesi & Escludere la gravidanza prima di iniziare il trattamento \\
\hline \multirow[t]{3}{*}{ DMF } & Effetti gastrointestinali & $\begin{array}{l}\text { Procinetici, stomaco pieno, dieta ricca in grassi e titolazione più lenta del } \\
\text { farmaco }\end{array}$ \\
\hline & Flushing & Rassicurare il pz, valutare se assunzione preventiva di ASA \\
\hline & Linfopenia & $\begin{array}{l}\text { Esame basale e monitoraggio dopo } 1,3 \text { e ogni } 3 \text { mesi. Se lym }<500 \text { per }>6 \\
\text { mesi, interruzione DMF }\end{array}$ \\
\hline \multirow[t]{3}{*}{ Alemtuzumab } & Reazioni infusionali & $\begin{array}{l}\text { Pretrattamento con corticosteroidi, antistaminici e antipiretici } \\
\text { Se reazione durante infusione: rallentare velocità di infusione; se reazione impor- } \\
\text { tante interrompere il farmaco e somministrare antistaminici/steroidi e/o adrenalina }\end{array}$ \\
\hline & Infezioni & $\begin{array}{l}\text { Esame di screening per anti-VZV, HIV, epatite B e C, Pap test per HPV nelle } \\
\text { donne; monitoraggio clinico e trattamento precoce di tutte le infezioni. } \\
\text { Aciclovir } 200 \mathrm{mg} \times 2 / \text { die dal } 1^{\circ} \text { giorno di trattamento per almeno } 1 \text { mese }\end{array}$ \\
\hline & Malattie autoimmuni secondarie & $\begin{array}{l}\text { Esame basale e monitoraggio mensile di emocromo con FL, creatinina ed } \\
\text { esame urine; se disfunzione visita nefrologica immediata } \\
\text { Esame basale di TSH e Ab antirecettore TSH, quindi controllo trimestrale di } \\
\text { TSH, se disfunzione subito trattamento } \\
\text { Educazione del pz per riconoscimento di segni PTI, disfunzione tiroidea e } \\
\text { disfunzione renale, se presenti trattamento immediato }\end{array}$ \\
\hline
\end{tabular}

$\mathrm{Ab}$, anticorpi; ASA, acido acetilsalicilico; AST/ALT, aspartato transaminasi/alanina aminotransferasi; BAV, blocco atrioventricolare; bpm, battiti per minuto; DMF, dimetilfumarato; DMD, disease modifying drug; ECG, elettrocardiogramma; FANS, farmaci antinfiammatori non steroidei; FC, frequenza cardiaca; FE, frazione d'eiezione; FL, formula leucocitaria; FO, fundus oculi; FTY, fingolimod; GA, glatiramer acetato; GB, globuli bianchi; HPV, papillomavirus umano; IFN-beta, interferone beta; JCV, John Cunningham virus; LMA, leucemia mieloide acuta; lym, linfociti; MTX, mitoxantrone ev; NAT, natalizumab; OCT, tomografia oculare; PML, leucoencefalopatia multifocale progressiva; PTI, porpora trombocitopenica idiopatica; pz, paziente; RM, risonanza magnetica; STRATIFY, test per anticorpi anti-JCV; TBC, tubercolosi; TSH, thyroidstimulating hormone; VZV, varicella zoster virus. ${ }^{(1)} \mathrm{Il}$ farmaco va sospeso temporaneamente se ALT/AST sono $>5$ volte il limite superiore della norma (LSN). Se $>3$ volte LSN: vanno monitorati valori AST/ALT e, se valore confermato, ridurre o sospendere temporaneamente a seconda del farmaco. Se $>3$ volte LSN ma associato a riduzione albumina o allungamento PT o rialzo bilirubina: sospensione del farmaco. ${ }^{(2)}$ Vedere Fig. 1 per rischio di PML con NAT. 


\section{Interferone beta}

L'IFN $\beta$ è disponibile sotto due forme: IFN $\beta$-1a e IFN $\beta-1 b$, caratterizzati da differenze nella sequenza proteica e nella glicosilazione. L'IFN $\beta$-1a viene somministrato al dosaggio di $30 \mu \mathrm{g}$ una volta alla settimana per via intramuscolare (im; Avonex ${ }^{\circledR}$ ), oppure tre volte a settimana per via sc, al dosaggio di $22 \mu \mathrm{g}$ o $44 \mu \mathrm{g}\left(\right.$ Rebif $\left.^{\circledR}\right)$, mentre IFN $\beta-1 \mathrm{~b}$ viene dato al dosaggio di $250 \mu$ g per via sc a giorni alterni (Betaferon ${ }^{\circledR} /$ Extavia $^{\circledR}$ ). Inoltre è recente l'approvazione di una formulazione di IFN $\beta$-1a a lento rilascio (Plegridy ${ }^{\circledR}$ ), $125 \mu \mathrm{g}$ sc ogni 2 settimane [5-8].

Complessivamente, il trattamento con IFN è considerato sicuro e ben tollerato [3].

Gli eventi avversi (EA) più comuni sono i ben noti sintomi simil-influenzali presenti soprattutto nei primi mesi, ma che tendono poi a migliorare o a scomparire nel tempo [2, 3]. I sintomi possono essere ridotti o annullati con l'assunzione di analgesici o antipiretici prima o al momento dell'iniezione. Sono stati segnalati casi di linfopenia, neutropenia, leucopenia e anemia [5-9] e lo sviluppo di altre malattie autoimmuni, principalmente ipotiroidismo [10, 11]. Sono stati riportati casi isolati di danno epatico grave $[12,13]$ e di sindrome nefrosica [14, 15]. Inizialmente si è ritenuto che IFN causasse o peggiorasse la depressione, ma una successiva metanalisi ha messo in evidenza frequenze di depressione simili nei pazienti trattati o meno $[16,17]$.

Prima di iniziare la terapia è opportuno indagare la presenza in anamnesi di patologie disimmuni, epatiche, tiroidee o depressione.

Il monitoraggio con emocromo, enzimi epatici e creatinina andrà eseguito dopo 1, 3, 6, 9 e 12 mesi e quindi ogni 6 mesi durante la tp. In generale le anomalie di laboratorio possono essere controllate con riduzione del dosaggio o della frequenza di somministrazione dell'IFN. Il farmaco va sospeso temporaneamente se ALT/AST sono > 5 volte il limite superiore della norma (LSN) e ridotto se $>3$ volte LSN in due controlli successivi; solo dopo normalizzazione si può ritornare alla dose intera. Il TSH va eseguito dopo 6 mesi di trattamento e in seguito solo in caso di valori alterati o in presenza di segni clinici di ipo/ ipertiroidismo. L'IFN può indurre la produzione di anticorpi (Ab) neutralizzanti [18]. Il test per tali $\mathrm{Ab}$ anti-IFN non è attualmente universalmente raccomandato, ma è consigliato nel caso di attività clinica o neuroradiologica di malattia e nel caso di positività va considerata l'interruzione della tp [19].

\section{Glatiramer acetato}

GA (Copaxone ${ }^{\circledR}$ ) è un polimero di 4 aminoacidi somministrato al dosaggio di $20 \mathrm{mg} /$ die per via sc. Recentemente è stata approvata una nuova formulazione $40 \mathrm{mg}$ sc tre volte alla settimana. GA presenta la più bassa incidenza di EA fra gli immunomodulanti [20-22].

La ben nota reazione sistemica immediata post-iniezione, caratterizzata da flushing a livello viso e torace, palpitazioni e tachicardia si manifesta in circa il $10 \%$ dei pazienti in maniera occasionale e si autolimita. Pertanto il paziente deve esserne informato e rassicurato [4]. Recentemente sono stati riportati rari casi di tossicità epatica [23, 24].

GA è un farmaco sicuro nel lungo termine $[4,21]$. Nessun monitoraggio di laboratorio è richiesto durante la tp.

\section{Mitoxantrone}

Mitoxantrone (MTX; Novantrone ${ }^{\circledR}$ ) è un farmaco citotossico somministrato per via ev a dosaggi variabili di $10-12 \mathrm{mg} / \mathrm{m}^{2}$ e a intervalli variabili di 1-3 mesi $[25,26]$.

Numerosi sono gli EA di MTX; quelli strettamente correlati alla somministrazione, ossia la possibile comparsa di nausea e/o vomito nonostante il pretrattamento con antiemetici, per cui vanno consigliati dieta leggera e antiemetici anche dopo la somministrazione. Il paziente deve essere informato che l'urina può essere blu per alcuni giorni dopo l'infusione. Un altro EA include la perdita transitoria dei capelli $[27,28]$.

La riduzione del numero totale dei globuli bianchi (GB) e dei linfociti (lym), dovuta al meccanismo d'azione di MTX, va monitorata sia perché deve essere dimostrato che vi sia un'effettiva riduzione sia perché un'eccessiva 
riduzione può aumentare i rischi infettivi, fra cui prevalentemente infezioni delle vie urinarie e delle vie aeree. Casi di tossicità epatica sono riportati nel 15\% dei pazienti. Il monitoraggio degli EE è previsto 10-12 giorni dopo l'infusione per valutare l'entità dell'immunosoppressione a livello ematologico e, in base al livello dei $\mathrm{GB}$, valutare se occorra un aggiustamento della dose di MTX. Gli stessi EE vanno ripetuti pochi giorni prima della successiva somministrazione e il farmaco non andrà somministrato se: neutrofili $<1500 / \mathrm{mm}^{3}$, piastrine $<50.000 / \mathrm{mm}^{3}$, AST/ALT $>3$ volte LSN [27].

Un limite all'utilizzo di MTX è la cardiotossicità dose-dipendente [riduzione della frazione di eiezione (FE) e insufficienza cardiaca congestizia sinistra] [29-31]. Tale complicanza si può sviluppare durante o anche dopo il termine della tp con MTX. Prima di ogni somministrazione si deve eseguire un ECG ed è raccomandato il monitoraggio con ecocardiogramma e valutazione della frazione di eiezione (FE) da eseguirsi prima del primo ciclo e poi ogni 3 mesi. Se FE $<50 \%$ o se si verifica una riduzione significativa della stessa, il MTX non va somministrato. Occorrono controlli ecocardiografici annuali anche dopo la cessazione di MTX.

Diversi studi hanno evidenziato un rischio di sviluppare leucemia mieloide acuta (LMA) durante o dopo la tp con MTX e in particolare uno studio italiano ha evidenziato un'incidenza di $0,93 \%$, per cui la dose cumulativa massima consigliata è $60 \mathrm{mg} / \mathrm{m}^{2}$ [32]. Anche dopo la sospensione di MTX permane il rischio di LMA e quindi il monitoraggio degli EE va eseguito ogni 3-4 mesi per 6 anni [33].

MTX può causare sterilità. Nelle donne di età $<35$ anni può causare amenorrea transitoria, mentre in quelle $>35$ anni può indurre menopausa precoce [28].

La gravità degli EA limita l'uso di MTX a pochi cicli, da effettuarsi come induzione per una fase di malattia molto attiva per poi proseguire con una tp di mantenimento [34, 35].

\section{Natalizumab}

Natalizumab (NAT; Tysabri ${ }^{\circledR}$ ) è un anticorpo monoclonale ricombinante umanizzato somministra- to per via ev [36-38] al dosaggio di $300 \mathrm{mg}$ mensili. NAT è ben tollerato. Gli EA più frequenti (>10\%) sono: febbre, cefalea, astenia, artralgie, vertigini; globalmente i pazienti riferiscono un'ottima qualità di vita (QoL) [39]. Il rischio infettivo, a eccezione del rischio di leucoencefalopatia multifocale progressiva (PML) descritto più avanti, è basso tranne che per una maggiore frequenza di riattivazioni erpetiche [40].

A livello di EE, si può verificare un rialzo di AST/ALT e bilirubina, tipicamente entro 1 settimana dalla $1^{\mathrm{a}}$ infusione, ma si può verificare anche tardivamente e può richiedere la sospensione del farmaco. Gli EE vanno ripetuti mensilmente prima di ogni somministrazione di NAT per i primi 6-12 mesi, poi ogni 3-6 mesi [37].

Si possono verificare le classiche reazioni infusionali durante o entro 2 ore dall'infusione; nel primo caso si risolvono rallentando la velocità di infusione. È però importante distinguerle dalla reazione da ipersensibilità (4\%), che può variare da un'orticaria fino a una reazione anafilattica $(<1 \%)$. In questi casi è necessario sospendere immediatamente l'infusione, somministrare farmaci antistaminici, steroidi e/o adrenalina. Tali reazioni da ipersensibilità si verificano più frequentemente dalla $2^{\mathrm{a}}$ infusione e soprattutto in pazienti positivi agli Ab anti-NAT $[37,38]$.

Circa il 9\% dei pazienti sviluppa, generalmente nei primi 3 mesi di trattamento, Ab neutralizzanti. La presenza di tali Ab può essere transitoria, mentre nel $6 \%$ si ha una positività persistente associata a una maggiore incidenza di reazioni allergiche e anche a una mancata efficacia del trattamento; in tali pazienti la tp andrebbe interrotta $[37,38]$.

Il rischio più grave correlato alla tp con NAT è la PML, infezione opportunistica dovuta alla riattivazione del JC virus (Figura 1) [41]. La condizione sine qua non per sviluppare la PML è avere avuto il contatto con il JCV e ciò si dimostra con il test per gli Ab-anti JCV [42]. La prevalenza della sieropositività nei pazienti con SM si aggira attorno al 58\% [43]. La presenza degli $\mathrm{Ab}$ è un fattore di rischio indipendente che va integrato con altri fattori quali la durata del trattamento (oltre i 24 mesi) e il precedente uso di immunosoppressori [44-47]. Nei pazienti sieronegativi il 


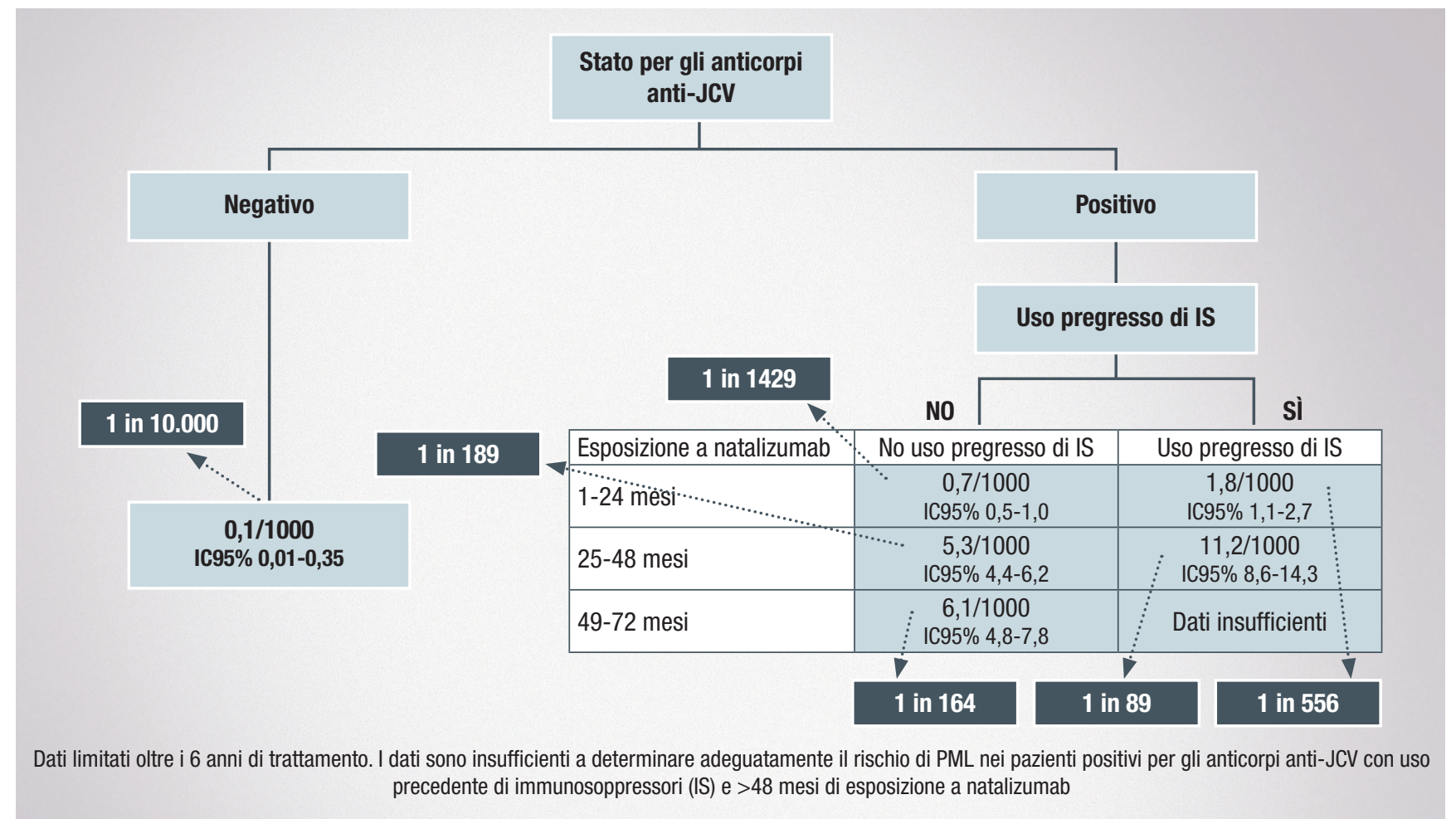

Figura 1. Algoritmo dei livelli di rischio di leucoencefalopatia multifocale progressiva (PML) nei pazienti trattati con natalizumab (non considerando il JC virus antibody Index), aggiornato al dicembre 2014. (Fonte: http://multiple-sclerosis-research.blogspot.com/2015/01/ clinicspeak-natalizumab-pml-update-q4.html. Downloaded November 30, 2015).

test va ripetuto ogni 6 mesi in quanto vi può essere una sieroconversione. Se il paziente è sieropositivo dopo 18-24 cicli di trattamento, va discusso con il paziente il rapporto rischio/beneficio della prosecuzione del trattamento. Per i pazienti che continuano il trattamento, sono necessarie RM encefalo ogni 3-4 mesi al fine di porre una diagnosi preclinica di PML. I pazienti che sospendono NAT devono essere informati del rischio di riattivazione della malattia dal $2^{\circ}$ all' $8^{\circ}$ mese post-sospensione, con possibile accumulo di disabilità $[48,49]$. Non esiste una cura per la PML e quindi le azioni fondamentali da intraprendere al minimo sospetto sono: interruzione immediata di NAT, RM encefalo con gadolinio, eseguire una puntura lombare per la ricerca di JCV mediante reazione polimerasica a catena (PCR) [se negativa non esclude PML]. Occorre uno stretto monitoraggio clinico e di risonanza magnetica (RM) [inizialmente anche quindicinale] al fine di monitorare l'evoluzio- ne della PML e diagnosticare tempestivamente l'eventuale comparsa di una sindrome infiammatoria da immunoricostituzione (IRIS), evento molto grave che richiede un'immediata somministrazione di steroidi [41, 47].

La plasmaferesi, effettuata al fine di rimuovere velocemente natalizumab dal sangue, è tema di revisione in quanto sembra possa accelerare la comparsa e la gravità dell'IRIS.

\section{Fingolimod}

Fingolimod (FTY; Gylenia ${ }^{\circledR}$ ), immunosoppressore selettivo, è il primo trattamento orale per la SM [50-54], al dosaggio di $0,5 \mathrm{mg} /$ die.

I più comuni EA sono influenza, sinusite, cefalea, diarrea, dolore alla schiena, innalzamento degli enzimi epatici e tosse. Particolare attenzione è rivolta 
verso gli effetti cardiaci di FTY, che provoca una bradicardia transitoria nelle prime 6 ore di trattamento. Gli studi clinici hanno mostrato una frequenza di bradicardie sintomatiche nello $0,5 \%$ dei pazienti e il verificarsi di blocchi atrioventricolari di I grado nel $4,7 \%$ dei pazienti. Altri rischi osservati negli studi clinici includono un lieve aumento della pressione arteriosa $(3 \mathrm{mmHg}$ di aumento medio della pressione sistolica, $1 \mathrm{mmHg}$ di aumento medio della diastolica), diminuzione della funzione polmonare lieve e dose-dipendente, edema maculare nello $0,5 \%$ dei pazienti. Negli studi clinici il tasso globale di infezioni $(65,1 \%)$ riportato con la dose di $0,5 \mathrm{mg}$ è risultato simile a quello osservato con il placebo. Tuttavia le infezioni delle basse vie respiratorie, principalmente bronchiti e, in misura minore, infezioni erpetiche e polmoniti, sono risultate più frequenti nei pazienti trattati con FTY [55-57].

Prima di iniziare il trattamento il paziente deve eseguire obbligatoriamente il dosaggio di Ab-anti virus varicella zoster (VZV). In caso di negatività il paziente deve sottoporsi al vaccino per VZV. Rimane comunque durante il trattamento un rischio di riattivazione di VZV [58]. In tal caso, il trattamento antivirale va iniziato immediatamente e a dosi piene e, se l'infezione è grave ossia multimetamerica, FTY deve essere sospeso.

Prima di iniziare FTY il paziente deve eseguire un ECG per escludere la presenza di disturbi del ritmo ed è consigliata, ma non presente in scheda tecnica, una visita cardiologica per escludere una cardiopatia. Infatti alla $1^{a}$ somministrazione si può verificare una bradicardia sintomatica solo nello 0,6\% dei casi, BAV di I grado nel 4,7\% e BAV di II grado nello 0,2\%, rendendo necessario un monitoraggio ECG continuo per 6 ore e controllo dei parametri vitali una volta all'ora $[53-57,59]$. Se al termine delle 6 ore la frequenza cardiaca è la più bassa di tutta l'osservazione, il monitoraggio cardiaco va prolungato per altre 2 ore. Inoltre è necessario prolungare l'osservazione fino al mattino seguente nella struttura ospedaliera in caso di: FC $<45$ bpm; BAV di III grado; BAV di II grado di nuova insorgenza e intervallo QTc $\geq 500 \mathrm{msec}$. Inoltre, l'intera procedura di monitoraggio va ripetuta se FTY viene interrotto per 1 giorno durante le prime 2 settimane di trattamento o per 7 giorni durante la settimana 3 e 4 o per più di 2 settimane dopo 1 mese di trattamento. Al paziente può essere consigliata una visita oculistica basale prima di iniziare FTY, che è invece mandatoria in pazienti con diabete mellito o anamnesi di uveite al fine di valutare fundus oculi (FO) ed escludere presenza o propensione a edema della macula. Inoltre tale visita va eseguita 3-4 mesi dopo l'inizio della terapia in quanto edema della macula si può verificare in questo intervallo temporale. La valutazione del $\mathrm{FO}$ inoltre va ripetuta in qualsiasi momento se il paziente lamenta riduzione dell'acuità visiva [60]. Nel caso di comparsa di edema maculare la terapia va sospesa e lo stesso scompare spontaneamente dopo l'interruzione.

A livello ematologico una leucopenia con linfopenia è un evento atteso per il meccanismo d'azione di FTY. Il controllo di emocromo con formula leucocitaria (FL) e di AST/ALT va eseguito dopo 1 mese e quindi ogni 3 mesi. Il farmaco va sospeso in caso di conta di $\mathrm{GB}<2000 / \mathrm{mm}^{3}$ o di $\mathrm{Lym}<200 / \mathrm{mm}^{3}$. In caso di rialzo di AST/ALT: se valore persiste $>5$ volte LSN, il farmaco va interrotto e se $>3$ volte LSN confermato, va valutata la sospensione in base ai valori di bilirubina, PT e albumina [55-57].

Sono segnalati inoltre possibile incremento di colesterolo/trigliceridi e rialzo della pressione arteriosa (PA) e pertanto è utile un monitoraggio periodico [57].

\section{Teriflunomide}

Teriflunomide (Aubagio ${ }^{\circledR}$ ) è un immunosoppressore selettivo, che inibisce le cellule $\mathrm{T}$ attivate, e che viene somministrato per os al dosaggio di $14 \mathrm{mg} /$ die [61-65]. Gli EA più frequenti sono disturbi gastrointestinali (nausea, diarrea), assottigliamento o perdita dei capelli e incremento degli enzimi epatici, che tendono a risolversi in corso di trattamento. Altri EA comuni sono riduzione della conta dei GB, lieve aumento del rischio infettivo, soprattutto delle alte vie respiratorie, cefalea, aumento dei valori di PA, artralgie [62, 66]. Negli studi registrativi sono stati segnalati due casi d'infezione op- 
portunistica, ossia una tubercolosi (TBC) intestinale e un'epatite da citomegalovirus [62, 67].

Il monitoraggio del farmaco prevede, prima di iniziare il trattamento, un controllo degli enzimi epatici, dell'emocromo con FL e conta piastrinica e una valutazione della PA e, in corso di trattamento, un controllo degli enzimi epatici e bilirubina ogni 2 settimane per i primi 6 mesi e quindi ogni 8 settimane; inoltre un controllo di emocromo con FL e creatinina dopo 1, 3, 6 mesi e quindi ogni 3-6 mesi. Se il livello di AST/ALT è >3 volte LSN in controlli successivi, il trattamento deve essere interrotto. Al monitoraggio di laboratorio si deve aggiungere il controllo della PA.

Teriflunomide è controindicata in gravidanza e in corso di allattamento, nei pazienti con grave danno epatico, con infezione in atto grave, con funzionalità del midollo osseo compromessa in modo significativo, con compromissione renale grave sottoposti a dialisi, in caso di grave ipoproteinemia e in caso di immunodeficienza.

Prima di iniziare il trattamento con teriflunomide il medico curante deve escludere una tubercolosi latente. Va ricordato ai pazienti che, se non eseguita la procedura di eliminazione con colestiramina, teriflunomide rimarrà nel sangue mediamente 8 mesi, fino a un massimo di 2 anni $[68,69]$.

\section{Dimetilfumarato}

Dimetilfumarato (DMF; Tecfidera ${ }^{\circledR}$ ), somministrato per os al dosaggio di $240 \mathrm{mg}$ due volte al giorno, ha una duplice azione antinfiammatoria e neuroprotettiva [70-72].

Gli EA più comuni associati al trattamento sono stati infezioni, soprattutto delle alte vie respiratorie, e rialzo degli enzimi epatici; più frequenti e talora causa di sospensione del trattamento sono il flushing ossia rossore, soprattutto al volto e parte alta torace (30-40\% dei pazienti), ed eventi gastrointestinali (GI) quali diarrea, nausea, dolore addominale soprattutto nei primi 2-3 mesi di trattamento (GI sino al 20$25 \%$ dei pazienti) [71, 72]. Per il flushing, verrebbe consigliata l'assunzione di ASA 30 minuti prima di
DMF [73], ma non è pratica comune sia per la variabilità del sintomo che per i possibili EA da ASA. Per i disturbi GI la pratica clinica suggerisce l'uso di procinetici e/o antiacidi. L'assunzione di DMF dopo un pasto abbondante ricco in grassi e forse una titolazione più lenta del farmaco possono ridurre i disturbi GI [72].

DMF può causare linfopenia nel primo anno di trattamento con una riduzione dei lym ancora nella norma nel 30\% dei pazienti e $<500 \mathrm{lym} / \mathrm{mm}^{3}$ nel 5\% dei pazienti. Il monitoraggio prevede un controllo dell'emocromo con FL e AST/ALT dopo 1 e 3 mesi di trattamento e successivamente ogni 3 mesi [71, 72]. Recentemente sono stati segnalati tre casi di PML in pazienti con SM trattati con DMF e con linfopenia severa persistente. Pertanto, l'European Medicines Agency (EMA) e l'Agenzia Italiana del Farmaco (AIFA) hanno dato indicazioni di sospendere il farmaco se i lym permangono $<500 / \mathrm{mm}^{3}$ per più di 6 mesi $[74,75]$.

\section{Alemtuzumab}

Alemtuzumab (Lemtrada ${ }^{\circledR}$ ) è un anticorpo monoclonale somministrato per via ev al dosaggio di $12 \mathrm{mg} / \mathrm{die}$ per 5 giorni al $1^{\circ}$ ciclo di trattamento e per 3 giorni al $2^{\circ}$ ciclo previsto dopo 1 anno. È un farmaco che depleta i lym B e T [76-79].

Le reazioni infusionali si verificano nel $90 \%$ dei pazienti e si manifestano soprattutto nel primo giorno di infusione (rash, cefalea, febbre, stanchezza, nausea) e sono gravi in $<3 \%$ casi $[78,79]$. Per ridurre l'entità di tali reazioni i pazienti vengono pretrattati con metilprednisolone ev (500-1000 mg per 3 o 5 giorni) e inoltre con antistaminici e antipiretici ogni giorno dell'infusione. Le infezioni compaiono fino nel $70 \%$ dei pazienti soprattutto nel primo mese (nel 3\% gravi): infezioni delle vie aeree superiori, infezioni delle vie urinarie, influenza, infezioni virali erpetiche [78, 79]. Per tale motivo dal primo giorno della prima infusione è necessario iniziare la profilassi con aciclovir (200 $\mathrm{mg} \times 2$ /die per os) per il primo mese. Vi sono state anche infezioni da papillomavirus umano (HPV) nel 2\% dei casi e infezioni fungine localizzate. 
Nel 20\% dei pazienti si possono manifestare fino a 5 anni dopo il trattamento patologie autoimmuni secondarie: nel 36\% disturbi della tiroide, in meno del $2 \%$ porpora trombocitopenica autoimmune (ITT), nello $0,3 \%$ nefropatie autoimmuni [80].

Il monitoraggio del farmaco prevede, prima di iniziare la tp: EE di base, TSH e Ab-antirecettore TSH, screening TBC, epatite B e C, Ab anti-VZV, HIV e inoltre pap test con HPV per le donne. Successivamente è richiesto uno stretto monitoraggio: emocromo (attenzione al valore delle piastrine), creatininemia e analisi delle urine con conta delle cellule urinarie una volta al mese e TSH ogni 3 mesi per i successivi 5 anni dopo il primo ciclo di trattamento. Oltre ai controlli ematologici è necessario educare i pazienti a riconoscere sintomi e segni di PTI, di disfunzione tiroidea e di disfunzione renale in modo da poter intervenire precocemente nel caso di alterazioni a livello ematologico e/o sintomatologico e instaurare immediatamente il trattamento adeguato [81].

\section{Conclusioni}

Le opzioni di trattamento per la SM si sono ampliate enormemente e continueranno a espandersi nel prossimo futuro.

Per i nuovi farmaci occorre uno stretto monitoraggio degli EA già evidenziati nei trials registrativi, per confermarne la frequenza e l'intensità e per segnalare EA non ancora riportati. Il neurologo deve conoscere gli EA dei vari farmaci e la loro gestione e deve illustrare al paziente gli stessi sia per permettere una buona QoL sia per evitare EA gravi.

Inoltre il numero elevato di trattamenti per la SM ha reso possibile un trattamento precoce e personalizzato ("taylored therapy") in cui un'attenta valutazione del rapporto rischio-beneficio è parte dell'algoritmo decisionale per il singolo paziente.

Uno strumento utile per la scelta e il monitoraggio della terapia sarebbe una sorta di "carta d'identità" del paziente comprensiva di uno screening infettivologico soprattutto per epatite B, C, per TBC, per anticorpi-anti-VZV, da raccogliere prima di iniziare un qualsiasi trattamento.
Inoltre sarebbe utile consigliare al paziente di eseguire il vaccino per l'epatite $\mathrm{B}$ (se non già eseguito), per l'epatite A (paziente giovane che viaggia), per HPV nelle donne e in quella piccola percentuale di pazienti sieronegativi per Ab-anti-VZV per la varicella.

\section{Disclosures}

Conflicts of interest: The author declares she has received honoraria for speaking during meeting congress and for partecipating to advisory board from Biogen Idec, Merck-Serono, Novartis, SanofiGenzyme.

\section{Bibliografia}

1. Greenberg BM, Khatri BO, Kramer JF. Current and emerging multiple sclerosis theraupeutics. Continuum. 2010;16:58-77.

2. Gold R, Rieckmann P, Chang P, et al. The long-term safety and tolerability of high-dose interferon beta-1a in relapsing-remitting multiple sclerosis: 4-year data from the PRISMS study. Eur J Neurol. 2005;12(8):649-56

3. Kappos L, Traboulsee A, Constantinescu C, et al. Long term subcutaneous interferon beta-1a therapy in patients with relapsing-remitting MS. Neurology. 2006;67(6):944-53.

4. Korczyn AD, Nisipeanu P. Safety profile of copolymer 1: analysis of cumulative experience in the United States and Israel. J Neurol. 1996;243(4 Suppl. 1):S23-6.

5. IFN beta Multiple Sclerosis Study Group and the University of British Columbia MS/MRI Analysis Group. Interferon beta-1b in the treatment of multiple sclerosis: final outcome of the randomized controlled trial. Neurology. 1995;45:1277-85.

6. Jacobs LD, Cookfair DL, Rudick RA, et al. Intramuscular interferon beta-1a for disease progression in relapsing multiple sclerosis. Ann Neurol. 1996;39:285-94.

7. Kieseier BC, Arnold DL, Balcer LJ, et al. Peginterferon beta-1a in multiple sclerosis: 2 year results from ADVANCE. Mult Scler. 2015;21(8):1025-35.

8. PRISMS (Prevention of Relapses and Disability by Interferon beta-1a Subcutaneously in Multiple Sclerosis) Study Group. Randomised double-blind placebo-controlled study of interferon beta-1a in relapsing/remitting multiple sclerosis. Lancet. 1998;352:1498-504.

9. PRISMS Study Group and the University of British Columbia MS/ MRI Analysis Group. PRISMS-4: long-term efficacy of interferon-beta-1a in relapsing MS. Neurology. 2001;56:1628-36.

10. Caraccio N, Dardano A, Manfredonia F, et al. Long-term follow-up of 106 multiple sclerosis patients undergoing interferon-beta 1a or 1b therapy: predictive factors of thyroid disease development and duration. J Clin Endocrinol Metab. 2005;90:4133-7.

11. Durelli L, Ferrero B, Oggero A, et al.; Betaferon Safety Trial Study Group. Thyroid function and autoimmunity during interferon be- 
ta-1b treatment: a multicenter prospective study. J Clin Endocrinol Metab. 2001;86:3525-32.

12. Pietrosi G, Mandala L, Vizzini GB, et al. Fulminant hepatic failure and autoimmune disorders in patient with multiple sclerosis on interferon beta 1a: a fatal combination? Transpl Int. 2008;21(5):502-4.

13. Montero JL, Cerezo A, Fraga E, et al. Acute liver failure in a patient with multiple sclerosis treated with interferon-beta. Mult Scler. 2007;13(6):820.

14. Wallbach M, Gröne HJ, Kitze Betral. Nephrotic syndrome in a multiple sclerosis patient receiving long-term interferon beta therapy Am J Kidney Dis. 2013;61(5):786-9.

15. Kumasaka R, Nakamura N, Shirato K, et al. Nephrotic syndrome associated with interferon-beta-1b therapy for multiple sclerosis. Clin Exp Nephrol. 2006;10(3):222-5.

16. Mohr DC, Likosky W, Dwyer P, et al. Course of depression during the initiation of interferon beta-1a treatment for multiple sclerosis. Arch Neurol. 1999;56(10):1263-5.

17. Patten SB, Metz LM. Interferon beta-1a and depression in relapsing-remitting multiple sclerosis: an analysis of depression data from the PRISMS clinical trial. Mult Scler. 2001;7(4):243-8.

18. Hemmer B, Stüve $\mathrm{O}$, Kieseier $\mathrm{B}$, et al. Immune response to immunotherapy: the role of neutralising antibodies to interferon beta in the treatment of multiple sclerosis. Lancet Neurol. 2005;4:403-12.

19. Bertolotto A, Capobianco M, Amato MP, et al. Guidelines on the clinical use for the detection of neutralizing antibodies (NAbs) to IFN beta in multiple sclerosis therapy: report from the Italian Multiple Sclerosis Study group. Neurol Sci. 2014;35(2):307-16.

20. Johnson KP, Brooks BR, Cohen JA, et al. Copolymer 1 reduces relapse rate and improves disability in relapsing-remitting multiple sclerosis: Results of a phase III multicenter, double-blind, placebo-controlled trial. Neurology. 1995;45(7):1268-76.

21. Ford C, Goodman AD, Johnson K, et al. Continuous long-term immunomodulatory therapy in relapsing multiple sclerosis: Results from the 15-year analysis of the US prospective open-label study of glatiramer acetate. Mult Scler. 2010;16(3):342-50.

22. Khan O, Rieckmann P, Boyko A, et al.; GALA Study Group. Three times weekly glatiramer acetate in relapsing-remitting multiple sclerosis. Ann Neurol. 2013;73(6):705-13.

23. Subramaniam K, Pavli P, Llewellyn H, Chitturi S. Glatiramer acetate induced hepatotoxicity. Curr Drug Saf. 2012;7:186-8.

24. Arruti M, Castillo-Triviño T, de la Riva P, et al. Autoimmune hepatitis in a patient with multiple sclerosis under treatment with glatiramer acetate. Rev Neurol. 2012;55:190-2.

25. Fidler JM, De Joy SQ, Gibbons JJ Jr. Selective immunomodulation by the antineoplastic agent mitoxantrone. I. Suppression of B lymphocyte function. J Immunol. 1986;137(2):727-32.

26. Watson CM, Davison AN, Baker D, et al. Suppression of demyelination by mitoxantrone. Int J Immunopharmacol. 1991;13(7):923-30.

27. Cohen BA, Mikol DD. Mitoxantrone treatment of multiple sclerosis: safety considerations. Neurology. 2004;63:S28-32.
28. Le Page E, Leray E, Edan G, et al. Long-term safety profile of mitoxantrone in a French cohort of 802 multiple sclerosis patients: a 5-year prospective study. Mult Scler. 2011;17(7):867-75

29. Gbadamosi J, Munchau A, Weiller C, et al. Severe heart failure in a young multiple sclerosis patient. J Neurol. 2003;250:241-2.

30. Goffette S, van Pesch V, Vanoverschelde JL, et al. Severe delayed heart failure in three multiple sclerosis patients previously treated with mitoxantrone. J Neurol. 2005;252:1217-22.

31. Avasarala JR, Cross AH, Clifford DB, et al. Rapid onset mitoxantrone-induced cardiotoxicity in secondary progressive multiple sclerosis. Mult Scler. 2003;9(1):59-62.

32. Martinelli V, Cocco E, Capra R, et al. Acute myeloid leukemia in Italian patients with multiple sclerosis treated with mitoxantrone. Neurology. 2011;77(21):1887-95.

33. Kingwell E, Koch M, Leung B, et al. Cardiotoxicity and other adverse events associated with mitoxantrone treatment for MS. Neurology. 2010;74:1822-6.

34. Cocco E, Marrosu MG. The current role of mitoxantrone in the treatment of multiple sclerosis. Expert Rev Neurother. 2014;14(6):607-16.

35. Le Page E, Leray E, Taurin G, et al. Mitoxantrone as induction treatment in aggressive relapsing remitting multiple sclerosis: treatment response factors in a 5 year follow up observational study of 100 consecutive patients. J Neurol Neurosurg Psychiatry. 2008;79:52-6.

36. Engelhardt B, Kappos L. Natalizumab: targeting alpha 4-integrins in multiple sclerosis. Neurodegener Dis. 2008;5:16-22.

37. Polman $\mathrm{CH}$, O'Connor PW, Havrdova E, et al. AFFIRM Investigators. A randomized, placebo-controlled trial of natalizumab for relapsing multiple sclerosis. N Engl J Med. 2006;354:899-910.

38. Rudick RA, Stuart WH, Calabresi PA, et al. Natalizumab plus interferon beta-1a for relapsing multiple sclerosis. N Engl J Med. 2006;354:911-23.

39. Pucci E, Giuliani G, Solari A, et al. Natalizumab for relapsing remitting multiple sclerosis. Cochrane Database Syst Rev. 2011;(10):CD007621

40. Fine AJ, Sorbello A, Kortepeter C, Scarazzini L. Central nervous system herpes simplex and varicella zoster virus infections in natalizumab-treated patients. Clin Infect Dis. 2013;57:849-52.

41. Koralnik IJ. New insights into progressive multifocal leukoencephalopathy. Curr Opin Neurol. 2004;17(3):365-70.

42. Gorelik L, Lerner M, Bixler S, et al. Anti-JC virus antibodies: implications for PML risk stratification. Ann Neurol. 2010;68:295-303.

43. Olsson T, Achiron A, Alfredsson L, et al. Anti-JC virus antibody prevalence in a multinational multiple sclerosis cohort. Mult Scler. 2013;19(11):1533-8.

44. Kleinschmidt-DeMasters BK, Tyler KL. Progressive multifocal leukoencephalopathy complicating treatment with natalizum$\mathrm{ab}$ and interferon beta-1a for multiple sclerosis. N Engl J Med 2005;353(4):369-74.

45. Bloomgreen S, Richman S, Hotermans C. Risk of natalizumab asso- 
ciated progressive multifocal leukoencephalopathy. N Engl J Med. 2012;366:1870-80.

46. Clifford DB, De Luca A, Simpson DM, et al. Natalizumab-associated progressive multifocal leukoencephalopathy in patients with multiple sclerosis: lessons from 28 cases. Lancet Neurol. 2010;9(4):438-46.

47. Kleinschmidt-DeMasters BK, Miravalle A, Schowinsky J, et al. Update on PML and PML-IRIS occurring in multiple sclerosis patients treated with natalizumab. J Neuropathol Exp Neurol. 2012;71:604-17.

48. Sangalli F, Moiola L, Ferrè L, et al. Long-term management of natalizumab discontinuation in a large monocentric cohort of multiple sclerosis patients. Mult Scler Relat Disord. 2014;3(4):520-6.

49. Prosperini L, Annovazzi P, Capobianco, et al. Natalizumab discontinuation in patients with multiple sclerosis: Profiling risk and benefits at therapeutic crossroads. Mult Scler. 2015;21(13):1713-22.

50. Kappos L, Antel J, Comi G, et al. Oral fingolimod (FTY720) for relapsing multiple sclerosis. N Engl J Med. 2006;355(11):1124-40.

51. Cohen JA, Barkhof F, Comi G, et al. Oral fingolimod or intramuscular interferon for relapsing multiple sclerosis. N Engl J Med. 2010;362(5):402-15.

52. Kappos L, Radue EW, O'Connor P, et al. A placebo-controlled trial of oral fingolimod in relapsing multiple sclerosis. N Engl J Med. 2010;362(5):387-401.

53. Calabresi PA, Radue EW, Goodin D, et al. Safety and efficacy of fingolimod in patients with relapsing-remitting multiple sclerosis (FREEDOMS II): a double-blind, randomised, placebo-controlled, phase 3 trial. Lancet Neurol. 2014;13(6):545-56.

54. Kappos L, O'Connor P, Radue EW, et al. Long-term effects of fingolimod in multiple sclerosis: the randomized FREEDOMS extension trial. Neurology. 2015;84(15):1582-91.

55. Cohen JA, Chun J. Mechanisms of fingolimod's efficacy and adverse effects in multiple sclerosis. Ann Neurol. 2011;69(5):759-77.

56. Collins W, Cohen J, O'Connor P, et al. Long-term safety of oral fingolimod (FTY720) in relapsing multiple sclerosis: integrated analyses of phase 2 and 3 studies. Mult Scler. 2010;16(10 Suppl.):S295.

57. Kappos L, Cohen J, Collins W, et al. Fingolimod in relapsing multiple sclerosis: an integrated analysis of safety findings. Mult Scler Relat Disord. 2014;3(4):494-504.

58. Arvin AM, Wolinsky JS, Kappos L, et al. Varicella-zoster virus infections in patients treated with fingolimod: risk assessment and consensus recommendations for management. JAMA Neurol. 2015;72(1):31-9.

59. Di Marco JP, O'Connor P, Cohen JA, et al. First-dose effects of fingolimod: Pooled safety data from three phase 3 studies. Mult Scler Relat Disord. 2014;3(5):629-38.

60. Zarbin MA, Jampol LM, Jager RD, et al. Ophthalmic evaluations in clinical studies of fingolimod (FTY720) in multiple sclerosis. Ophthalmology. 2013;120(7):1432-9.

61. Confavreux C, Li DK, Freedman MS, et al. Long-term follow-up of a phase 2 study of oral teriflunomide in relapsing multiple sclerosis: safety and efficacy results up to 8.5 years. Mult Scler. 2012;18:1278-89.

62. Freedman MS, Wolinsky JS, Wamil B, et al. Teriflunomide added to interferon- $\beta$ in relapsing multiple sclerosis: a randomized phase II trial. Neurology. 2012;78:1877-85.

63. O'Connor P, Wolinsky JS, Confavreux C, et al. Randomized trial of oral teriflunomide for relapsing multiple sclerosis. N Engl J Med. 2011;365:1293-303.

64. O'Connor PW, Li D, Freedman MS, et al. A phase II study of the safety and efficacy of teriflunomide in multiple sclerosis with relapses. Neurology. 2006;66:894-900.

65. Confavreux C, O'Connor P, Comi G, et al. Oral teriflunomide for patients with relapsing multiple sclerosis (TOWER): a randomised, double-blind, placebo-controlled, phase 3 trial. Lancet Neurol. 2014;13(3):247-56

66. Freedman MS, Confavreux C, Comi G, et al. Hair thinning associated with teriflunomide therapy is manageable. Presented at the 4 th Cooperative Meeting of CMSC and ACTRIMS. 30 May-2 June 2012: San Diego, CA, USA.

67. Papadoupoulo A, Kappos L, Sprenger T. Safety of teriflunomide for the management of relapsing remitting multiple sclerosis. Expert Opin Drug Saf. 2015;14(5):749-59.

68. Miller A, Turpault S, Menguy-Vacheron F. Rapid elimination procedure of teriflunomide with cholestyramine or activated charcoal Presented at the 4th Cooperative Meeting of CMSC and ACTRIMS. 30 May-2 June 2012: San Diego, CA, USA.

69. Warnke C, Stüve O, Kieseier BC. Teriflunomide for the treatment of multiple sclerosis. Clin Neurol Neurosurg. 2013;115 Suppl 1:S90-4.

70. Linker RA, Lee D-H, Ryan S, et al. Fumaric acid esters exert neuroprotective effects in neuroinflammation via activation of the Nrf2 antioxidant pathway. Brain. 2011;134(3):678-92.

71. Fox RJ, Miller DH, Phillips JT, et al. Placebo-controlled phase 3 study of oral BG-12 or glatiramer in multiple sclerosis. N Engl J Med. 2012;367(12):1087-97.

72. Gold R, Kappos L, Arnold DL, et al. Placebo-controlled phase 3 study of oral BG-12 for relapsing multiple sclerosis. N Engl J Med. 2012;367(12):1098-107.

73. O'Gorman J, Russell HK, Li J, et al. Effect of aspirin pretreatment or slow dose titration on flushing and gastrointestinal events in healthy volunteers receiving delayed-release dimethyl fumarate. Clin Ther. 2015;37(7):1402-19.

74. Nieuwkamp DJ, Murk JL, van Oosten BW, et al. PML in a patient without severe lymphocytopenia receiving dimethyl fumarate. N Engl J Med. 2015;372(15):1474-6.

75. European Medicines Agency. 23/10/2015. Updated recommendations to minimize the risk of the rare brain infection PML with Tecfidera. htpp://www.ema.europa.eu/ema/index.jsp.

76. Ruck T, Bittner S, Wiendl H, Meuth SG. Alemtuzumab in multiple sclerosis: mechanism of action and beyond. Int J Mol Sci 2015;16(7):16414-39

77. Coles AJ, Cox A, Le Page E, et al. The window of therapeutic op- 
portunity in multiple sclerosis: evidence from monoclonal antibody therapy. J Neurol. 2006;253(1):98-108.

78. Cohen JA, Coles AJ, Arnold DL, et al. Alemtuzumab versus in terferon beta 1a as first line treatment for patients with relapsing-remitting multiple sclerosis: a randomised controlled phase 3 trial. Lancet. 2012;380(9856):1819-28.

79. Coles AJ, Twyman CL, Arnold D, et al. Alemtuzumab for patients with relapsing multiple sclerosis after disease-modi- fying therapy: a randomised controlled phase 3 trial. Lancet. 2012;380(9856):1829-39.

80. Cossburn M, Pace AA, Jones J, et al. Autoimmune disease after alemtuzumab treatment for multiple sclerosis in a multicenter cohort. Neurology. 2011;77(6):573-9.

81. Havrdova E, Horakova D, Kovarova I. Alemtuzumab in the treatment of multiple sclerosis: key clinical trial results and considerations for use. Ther Adv Neurol Disord. 2015;8(1):31-45. 